\title{
Neutron-induced fission cross section of ${ }^{240} \mathrm{Pu}$ from $0.5 \mathrm{MeV}$ to $3 \mathrm{MeV}$
}

\author{
P. Salvador-Castiñeira, ${ }^{*}$ T. Bryś, R. Eykens, F.-J. Hambsch, ${ }^{\dagger}$ A. Göök, A. Moens, S. Oberstedt, \\ G. Sibbens, D. Vanleeuw, and M. Vidali \\ European Commission, Joint Research Centre, Institute for Reference Materials and Measurements (JRC-IRMM), \\ Retieseweg 111, B-2440 Geel, Belgium \\ C. Pretel \\ Institute of Energy Technologies, Technical University of Catalonia, Avenida Diagonal 647, E-08028 Barcelona, Spain
} (Received 10 April 2015; published 21 July 2015)

\begin{abstract}
${ }^{240} \mathrm{Pu}$ has recently been pointed out by a sensitivity study of the Organization for Economic Cooperation and Development (OECD) Nuclear Energy Agency (NEA) to be one of the isotopes whose fission cross section lacks accuracy to meet the upcoming needs for the future generation of nuclear power plants (GEN-IV). In the High Priority Request List (HPRL) of the OECD, it is suggested that the knowledge of the ${ }^{240} \mathrm{Pu}(n, f)$ cross section should be improved to an accuracy within 1-3\%, compared to the present 5\%. A measurement of the ${ }^{240} \mathrm{Pu}$ cross section has been performed at the Van de Graaff accelerator of the Joint Research Center (JRC) Institute for Reference Materials and Measurements (IRMM) using quasi-monoenergetic neutrons in the energy range from $0.5 \mathrm{MeV}$ to $3 \mathrm{MeV}$. A twin Frisch-grid ionization chamber (TFGIC) has been used in a back-to-back configuration as fission fragment detector. The ${ }^{240} \mathrm{Pu}(n, f)$ cross section has been normalized to three different isotopes: ${ }^{237} \mathrm{~Np}(n, f),{ }^{235} \mathrm{U}(n, f)$, and ${ }^{238} \mathrm{U}(n, f)$. Additionally, the secondary standard reactions were benchmarked through measurements against the primary standard reaction ${ }^{235} \mathrm{U}(n, f)$ in the same geometry. A comprehensive study of the corrections applied to the data and the associated uncertainties is given. The results obtained are in agreement with previous experimental data at the threshold region. For neutron energies higher than $1 \mathrm{MeV}$, the results of this experiment are slightly lower than the ENDF/B-VII.1 evaluation, but in agreement with the experiments of Laptev et al. (2004) as well as Staples and Morley (1998).
\end{abstract}

DOI: 10.1103/PhysRevC.92.014620

PACS number(s): 25.85.Ec

\section{INTRODUCTION}

Some of the designs under study for the new generation of nuclear power plants (GEN-IV) are developed to work using a fast-neutron energy spectrum. Nuclear data in this region are scarce and, most of the time, their spread is large. Additionally, most data sets available date from the 1970s. To group all the specific needs for the most viable GEN-IV nuclear power plant designs, the Organization for Economic Cooperation and Development (OECD) Nuclear Energy Agency (NEA) issued in 2008 a sensitivity study [1]. Within this extended study, a list of nuclear data parameters of interest for fast reactors was published. In that list, not only improved accuracy of several cross sections of actinides were requested, but also some important nuclear parameters of structural materials present in such reactors. Specifically, the neutron-induced fission cross section of ${ }^{240} \mathrm{Pu}$ was listed to be improved to an accuracy within 1-3\%, compared to the present $5 \%$.

\footnotetext{
*Also at Institute of Energy Technologies, Technical University of Catalonia, Avda. Diagonal 647, E-08028 Barcelona, Spain. Currently at National Physical Laboratory, Hampton Road, Teddington, Middlesex TW11 0LW, United Kingdom.

†Franz-Josef.Hambsch@ec.europa.eu

Published by the American Physical Society under the terms of the Creative Commons Attribution 3.0 License. Further distribution of this work must maintain attribution to the author(s) and the published article's title, journal citation, and DOI.
}

The Accurate Nuclear Data and Energy Sustainability (ANDES) Collaboration [2] had the objective to address some of the data needs for the design of fast reactors. This project was enrolled in the collaboration to determine with an unprecedented accuracy the neutron-induced fission cross section of ${ }^{240,242} \mathrm{Pu}$. In this paper, the results of the ${ }^{240} \mathrm{Pu}(n, f)$ cross section measurements are being presented, while the results for the ${ }^{242} \mathrm{Pu}(n, f)$ cross section will be provided in a forthcoming publication.

Several data sets are available in the Experimental Nuclear Reaction Data Library (EXFOR) [3]. In Fig. 1 the most relevant experiments in the energy range of this measurement are plotted together with the most recent evaluations (for clearness not all data points are shown). The fission threshold of ${ }^{240} \mathrm{Pu}(n, f)$ is well determined. In the case of the plateau region there is a discrepancy of $7 \%$ between the newest data of Tovesson et al. (2009) [4] and the rest of the data sets [5-10]. In the same way, the JEFF 3.1 evaluation [11], obtained prior to Tovesson's experiment via a trial and error procedure of two codes, is 5\% higher than the ENDF/B-VII.1 [12], mainly obtained by relying on the data set of Staples and Morley (1998) [9]. The JENDL 4.0 [13] evaluation is obtained after performing a simultaneous fit of any subset of data later than 1960 of ${ }^{233,235,238} \mathrm{U}(n, f)$ and ${ }^{239,240,241} \mathrm{Pu}(n, f)$. Additionally, any of the experiments presented have reached an uncertainty below 3\%, taking into account that, in many cases, the uncertainty of the reference cross section is not considered.

In order to minimize uncertainties and bring additional information compared to that already available in the literature, 


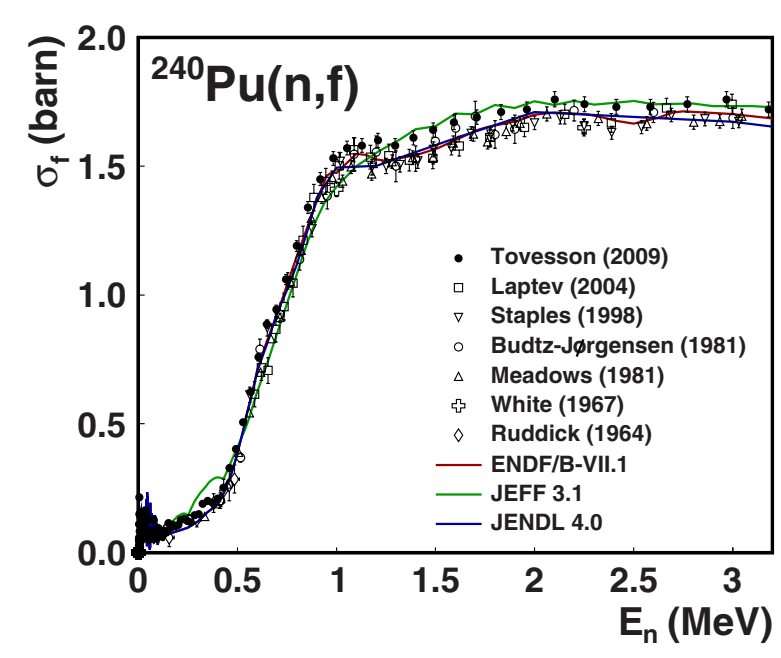

FIG. 1. (Color online) Summary of the most relevant experiments performed of the neutron-induced fission cross section of ${ }^{240} \mathrm{Pu}$ compared with current evaluations. The evaluations chosen are ENDF/B-VII.1 [12], JEFF 3.1 [11] and JENDL 4.0 [13]. The experimental data shown are Ruddick and White (1964) [5] (open diamonds), White (1967) [6] (open crosses), Meadows (1981) [7] (open triangle up), Budtz-Jørgensen and Knitter (1981) [8] (open circles), Staples and Morley (1998) [9] (open triangles down), Laptev et al. (2004) [10] (open squares), and Tovesson et al. (2009) [4] (full circles). Selected data are shown for visibility of the plot. Data found as a ratio of ${ }^{235} \mathrm{U}$ were normalized to the ENDF/B-VII.1 evaluation of this isotope. Further explanation is given in the text.

the experiment presented here used as reference cross sections ${ }^{237} \mathrm{~Np}(n, f),{ }^{238} \mathrm{U}(n, f)$, and ${ }^{235} \mathrm{U}(n, f)$. The cross section of ${ }^{235} \mathrm{U}(n, f)$ is considered a primary standard, the one of ${ }^{238} \mathrm{U}(n, f)$ a secondary standard from $2 \mathrm{MeV}$ onwards, and the one of ${ }^{237} \mathrm{~Np}(n, f)$ is not considered a standard. For this reason, the ${ }^{237} \mathrm{~Np}(n, f)$ and ${ }^{238} \mathrm{U}(n, f)$ cross sections were measured relative to the ${ }^{235} \mathrm{U}(n, f)$ cross section. Furthermore, the use of modern digital electronics was a key point to the complete understanding of the experimental data when using high $\alpha$-active targets such as ${ }^{240} \mathrm{Pu}$.

\section{EXPERIMENTAL SETUP}

\section{A. The Van de Graaff accelerator (JRC-IRMM)}

The Van de Graaff accelerator (VdG) of the JRC-IRMM was used to produce neutrons. At the $\mathrm{VdG}$ protons, deuterons or $\alpha$ particles are accelerated through a potential difference to impinge onto a neutron producing target. Different reactions are used to generate quasi-monoenergetic neutrons. In the particular case of this experiment, protons were accelerated to produce neutrons using the reactions ${ }^{7} \mathrm{Li}(p, n){ }^{7} \mathrm{Be}(0.5 \leqslant$ $\left.E_{n} \leqslant 1.8 \mathrm{MeV}\right)$ and $\mathrm{T}(p, n)^{3} \mathrm{He}\left(1.6 \leqslant E_{n} \leqslant 3.0 \mathrm{MeV}\right)$. The neutron producing targets are placed at the end of the beam line and, in order to keep their temperature below a certain level, they are cooled using a flow system of water with a layer thickness of 1-3 mm. The effect of the water cooling system on the neutron energy has been studied in detail by means of Monte Carlo simulations and will be discussed below.

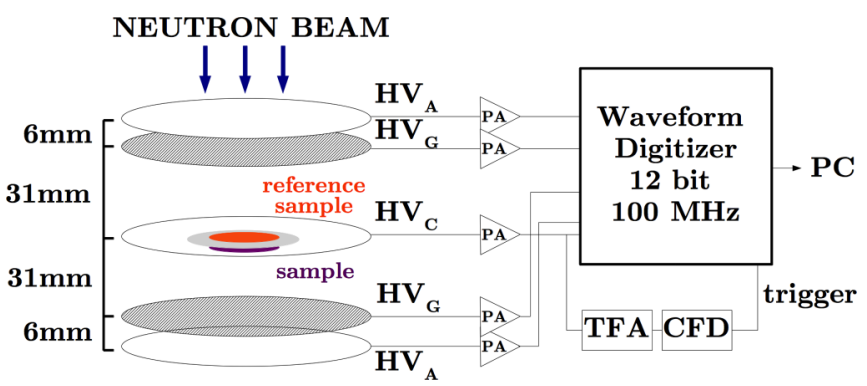

FIG. 2. (Color online) Scheme of the twin Frisch-grid ionization chamber with two samples in a back-to-back configuration followed by the digital electronics used for this experiment.

\section{B. Fission fragment detector}

A twin Frisch-grid ionization chamber (TFGIC) was used as fission fragment (FF) detector. This detector has been proven to provide outstanding results in FF characterization in many experiments, thanks to its characteristics: almost $4 \pi$ solid angle, radiation resistance, and high energy resolution. The type of TFGIC used in this work is described in Refs. [14,15]. In the case of this experiment the TFGIC was filled in a first stage with P10 (90\% $\left.\mathrm{Ar}+10 \% \mathrm{CH}_{4}\right)$ and later, with $\mathrm{CH}_{4}$. The main reason for using $\mathrm{CH}_{4}$ as counting gas was to increase the electron drift mobility to be able to correct for several $\alpha$ particles piling up on the recorded signal. An extended description of signals using one or the other gas was already published elsewhere [16]. The gas flow was about $50 \mathrm{ml} / \mathrm{min}(100 \mathrm{ml} / \mathrm{min})$ when using P10 $\left(\mathrm{CH}_{4}\right)$. A high voltage was applied to each electrode through charge sensitive preamplifiers. The anodes had a positive voltage of $1 \mathrm{kV}(1.5 \mathrm{kV})$, the grids were grounded, and the cathode had a negative voltage of $-1.5 \mathrm{kV}(-2.5 \mathrm{kV})$ when using $\mathrm{P} 10\left(\mathrm{CH}_{4}\right)$.

Figure 2 presents a sketch of the TFGIC together with the associated electronics scheme. The cathode-grid distance was chosen in order to fully stop the FFs before reaching the grid. For cross section experiments a common choice is to place in the cathode holder two samples, the sample under study and the reference sample, in a back-to-back geometry; in this way, a measurement of the neutron flux in the exact same place where the samples are may be avoided.

\section{Data acquisition (DAQ)}

The charge sensitive preamplifiers were fed into a 12 bit, $100 \mathrm{MHz}$ waveform digitizer (WFD). The WFD was triggered using a logical signal obtained from the cathode preamplifier after passing through a timing filter amplifier (TFA) and a constant fraction discriminator (CFD). An electronic threshold was set on the CFD to avoid $\alpha$ particles from the ${ }^{240} \mathrm{Pu}$ sample triggering the system. The signals were stored after the WFD in a computer for offline analysis using a data acquisition system (DAQ) developed at JRC-IRMM. The DAQ was built in $\mathrm{C}++$ using ROOT as framework $[17,18]$.

\section{Signal processing (DSP)}

Offline, the signals were analyzed using a digital signal processing code (DSP) developed at JRC-IRMM. The signals 
TABLE I. Description of the ${ }^{240} \mathrm{Pu}$ sample and the reference samples used $\left({ }^{237} \mathrm{~Np},{ }^{238} \mathrm{U}\right.$, and $\left.{ }^{235} \mathrm{U}\right)[19-21]$.

\begin{tabular}{|c|c|c|c|c|}
\hline & ${ }^{240} \mathrm{Pu}$ & ${ }^{237} \mathrm{~Np}$ & ${ }^{235} \mathrm{U}$ & ${ }^{238} \mathrm{U}$ \\
\hline Method & electrodeposition & vacuum deposition & vacuum deposition & vacuum deposition \\
\hline $\operatorname{Mass}^{\mathrm{a}}(\mu \mathrm{g})$ & $93(0.4 \%)$ & $390(0.3 \%)$ & $584(2 \%)$ & $577(0.4 \%)$ \\
\hline Areal density $\left(\mu \mathrm{g} / \mathrm{cm}^{2}\right)$ & $13.19(0.4 \%)$ & $308(0.3 \%)$ & $94.8(2 \%)$ & $81.7(0.4 \%)$ \\
\hline Backing & aluminum & stainless steel & stainless steel & transparent \\
\hline$\alpha$ activity $(\mathrm{MBq})$ & $0.780(0.4 \%)$ & $0.001(0.1 \%)$ & $265.7 \mathrm{~Bq}^{\mathrm{b}}(2 \%)$ & $7 \mathrm{~Bq}(0.5 \%)$ \\
\hline$\%{ }^{239} \mathrm{Pu}$ & 0.0144 & $0.2 \%{ }^{238} \mathrm{Pu}$ & $0.2 \%{ }^{234} \mathrm{U}$ & $<0.02 \%{ }^{234} \mathrm{U}$ \\
\hline$\%{ }^{240} \mathrm{Pu}$ & 99.8915 & & $0.03 \%{ }^{236} \mathrm{U}$ & \\
\hline$\%{ }^{241} \mathrm{Pu}$ & 0.0041 & & $0.3 \%{ }^{238} \mathrm{U}$ & \\
\hline$\%{ }^{242} \mathrm{Pu}$ & 0.0203 & & & \\
\hline$\%{ }^{244} \mathrm{Pu}$ & 0.0001 & & & \\
\hline
\end{tabular}

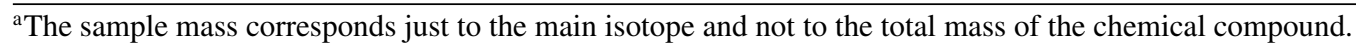

${ }^{\mathrm{b}}$ The sample activity of the ${ }^{235} \mathrm{U}$ sample considers the contribution of the ${ }^{234} \mathrm{U}$ and ${ }^{235} \mathrm{U}$ isotopes.

were treated with, basically, a baseline correction and a $\mathrm{CR}-\mathrm{RC}^{4}$ filter [15]. During this process the $\alpha$ particles, piled up in the baseline prior and after the FF event, were subtracted. The amplitude of each signal was correlated with the pulse height $(\mathrm{PH})$. The $\mathrm{PH}$ distributions were corrected for the grid inefficiency.

\section{E. Sample description}

Several samples were used within this experiment; all of them were made by the target preparation group of JRC-IRMM. An extended description is given in Table I. The mass of the ${ }^{240} \mathrm{Pu}$ sample was chosen to minimize its $\alpha$ activity without compromising the measuring time in the experiment. Nevertheless, its $0.8 \mathrm{MBq}$ of activity allowed signals of up to 7 $\alpha$ particles piling up together, that needed to be discriminated from low-energy FF signals. Ideally, to avoid solid angle corrections, the diameter of all the samples should be the same. Unfortunately, the only ${ }^{237} \mathrm{~Np}$ sample available had a spot size half of the one of the other samples. This made the ${ }^{237} \mathrm{~Np}$ sample much thicker than the rest. The purity of all the samples used was higher than $99.5 \%$, and the content of fissile isotope contaminants was smaller than $0.2 \%$. The mass of all samples was measured by means of low geometry $\alpha$ counting. The uncertainty reached was below $1 \%$ for all samples, except for the ${ }^{235} \mathrm{U}$. In that case, the $2 \%$ uncertainty is due to its complex $\alpha$ decay scheme. Additionally, the ${ }^{238} \mathrm{U}$ and the ${ }^{235} \mathrm{U}$ sample masses were remeasured using a single Frisch-grid ionization chamber. The results obtained were within the uncertainties of those obtained by the previous method.

\section{F. Homogeneity study}

Two methods of sample production were employed: molecular plating (for ${ }^{240} \mathrm{Pu}$ ) and vacuum deposition (for ${ }^{237} \mathrm{~Np},{ }^{235} \mathrm{U}$, and ${ }^{238} \mathrm{U}$ ). To study the homogeneity of the samples a qualitative and a quantitative technique were used. Initially, fluorescence images were taken of the ${ }^{240} \mathrm{Pu}$ and the ${ }^{237} \mathrm{~Np}$ samples [22]. In the case of the ${ }^{240} \mathrm{Pu}$ a higher $\alpha$ activity was found in the outer layer; in contrast, the ${ }^{237} \mathrm{~Np}$ sample presented a uniform distribution of the $\alpha$ activity on its surface. To quantify the mass increase seen in the outer layer of the ${ }^{240} \mathrm{Pu}$ sample, a low solid-angle $\alpha$-particle counting measurement was done. A mask with a $6 \mathrm{~mm}$ diameter was used to perform a measurement in the center of the mass and then several measurements in the outer layer. The increase of mass in the outer layer with respect to the center of the sample was of $11.4 \%$.

\section{G. Shielding}

The experimental setup was updated with a large paraffin- $\mathrm{B}_{4} \mathrm{C}$ shield when the ${ }^{235} \mathrm{U}$ sample was used as reference. The function of the shielding was to slow down (via the paraffin) the neutrons that were not interacting with the samples inside the TFGIC. Once those neutrons were in the target hall, if they were returning to the TFGIC, after scattering they would cross the paraffin again and then be captured in the $\mathrm{B}_{4} \mathrm{C}$ wall. Because of the large neutron capture cross section of ${ }^{10} \mathrm{~B}$, the probability that those thermalized neutrons reach the fissile ${ }^{235} \mathrm{U}$ will be minimized. Figure 3 shows the setup without (setup \#1) and with (setup \#2) shielding.

\section{CROSS SECTION MEASUREMENT}

Several measurements using a LiF neutron producing target and a TiT target to determine the following cross section ratios were performed: ${ }^{240} \mathrm{Pu}(n, f) /{ }^{237} \mathrm{~Np}(n, f)$, ${ }^{240} \mathrm{Pu}(n, f) /{ }^{238} \mathrm{U}(n, f)$, and ${ }^{240} \mathrm{Pu}(n, f) /{ }^{235} \mathrm{U}(n, f)$. Additionally, an effort was put into determining the cross section of ${ }^{237} \mathrm{~Np}(n, f)$ and the cross section of the secondary standard ${ }^{238} \mathrm{U}(n, f)$. To achieve these goals, the following ratios were measured too: ${ }^{237} \mathrm{~Np}(n, f) /{ }^{235} \mathrm{U}(n, f)$ and ${ }^{238} \mathrm{U}(n, f) /{ }^{235} \mathrm{U}(n, f)$.

The neutron-induced fission cross section was calculated as

$$
\begin{aligned}
\sigma_{\text {main }, 0}^{S}= & \frac{C_{S}}{C_{R}} \frac{F_{S}}{F_{R}}\left(\sum_{i} \frac{N_{i}^{R}}{N_{\text {main }}^{S}} \frac{\Phi_{0}^{R}}{\Phi_{0}^{S}} \sigma_{i, 0}^{R}+\sum_{i} \frac{N_{i}^{R}}{N_{\text {main }}^{S}} \frac{\Phi_{1}^{R}}{\Phi_{0}^{S}} \sigma_{i, 1}^{R}\right) \\
& -\sum_{i} \frac{N_{i}^{S}}{N_{\text {main }}^{S}} \frac{\Phi_{1}^{S}}{\Phi_{0}^{S}} \sigma_{i, 1}^{S}-\sum_{j} \frac{N_{j}^{S}}{N_{\text {main }}^{S}} \sigma_{j, 0}^{S}
\end{aligned}
$$



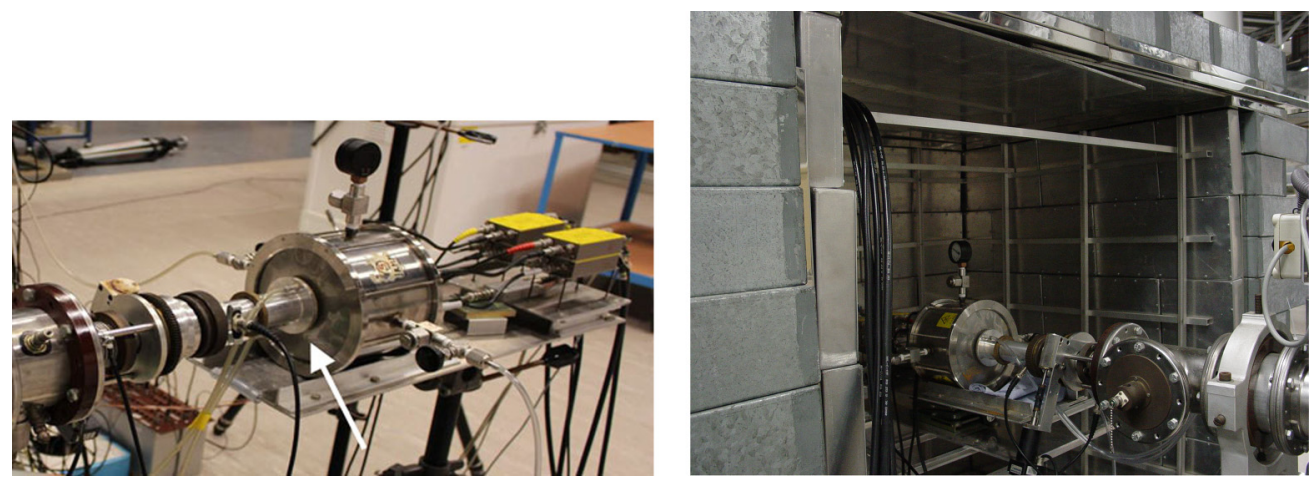

FIG. 3. (Color online) Left: setup configuration without the shielding (courtesy of D. Vanleeuw) (setup \#1). The arrow points to the cap of the neutron producing target. Right: setup configuration with the paraffin- $\mathrm{B}_{4} \mathrm{C}$ shielding (setup \#2).

where the index main represents the main isotope of the sample $(S), R$ refers to the reference sample, $C_{i}$ is the net count rate described as $C=\frac{C_{\text {total }}}{\epsilon}-\sum_{i} C_{S F, i}, F_{i}$ are the correction factors due to the background neutrons calculated using MCNP [23], $N$ are the numbers of atoms, $\Phi^{R} / \Phi^{S}$ is the ratio of neutron flux due to the difference of sample spot size and homogeneity, and the subindexes 0 and 1 refer to the neutron energy from a reaction to the nuclear ground state and to the neutron energy resulting from a reaction to the first excited state of the recoil nucleus of the reaction ${ }^{7} \mathrm{Li}(p, n)^{7} \mathrm{Be}$, respectively.

Hereafter an extended explanation of the applied corrections will be given (A), followed by a discussion on the sources of uncertainty (B), before the final cross section data is discussed (C).

\section{A. Corrections}

\section{Electronic threshold}

There are two experimental conditions that cause an incomplete $\mathrm{PH}$ distribution. One is the electronic threshold level applied to discard as many $\alpha$ particles as possible. The other is the thickness of the used sample: in a thicker sample FFs will loose more energy before entering into the counting gas, thus the $\mathrm{PH}$ distribution will be biased towards the low energy region. An example of both effects can be seen in Fig. 4. To correct for these effects a constant $\mathrm{PH}$ distribution towards zero $\mathrm{PH}$ is assumed. Thus, a linear extrapolation is done in this region. This correction was usually between $3 \%$ and $5 \%$ of the total detected counts. Nevertheless, when using the thickest sample $\left({ }^{237} \mathrm{~Np}\right)$ together with the most active sample $\left({ }^{240} \mathrm{Pu}\right)$, the correction to be applied to the ${ }^{237} \mathrm{~Np}$ sample was as large as $13.6 \%$.

\section{Spontaneous fission half-life}

The spontaneous fission rate of the ${ }^{240} \mathrm{Pu}$ sample was about 0.05 fissions/s. An extended study was performed earlier to determine the spontaneous fission half-life of this isotope, i.e., $T_{1 / 2, S F}=1.165 \times 10^{11} \mathrm{yr}(1.1 \%)$ [16]. This correction was important in the whole neutron energy range covered, since the fission rate for any single energy was never higher than 2-3 fissions/s. Around the fission threshold the correction exceeded $5 \%$.

\section{Self-absorption and efficiency}

The efficiency of a TFGIC used in a back-to-back configuration is nearly $2 \times 2 \pi$; if a FF enters the counting gas, it will always ionize the counting gas producing a signal on the electrodes. Therefore, the detection efficiency will be mainly deteriorated by the self-absorption of FFs inside the sample. In the case of $(n, f)$ reactions two other factors need to be included: the momentum transfer from the neutron to the fissioning nucleus and the anisotropic FF emissions; both of them depending on the incoming neutron energy. The equations used to calculate the efficiency were derived from Ref. [24],

$$
\begin{aligned}
& \epsilon=\left[1-\frac{t_{0}}{2 R}\left(1+2 \eta \frac{R}{t_{0}}\right)\left(1-\frac{a_{2}}{2}\right)\right] \text { downstream, } \\
& \epsilon=\left\{1-\frac{t_{0}}{2 R}\left[1-\eta\left(\frac{2 R}{t_{0}}-\eta \frac{R^{2}}{t_{0}^{2}}\right)\right]\left(1-\frac{a_{2}}{2}\right)\right\} \text { upstream, }
\end{aligned}
$$

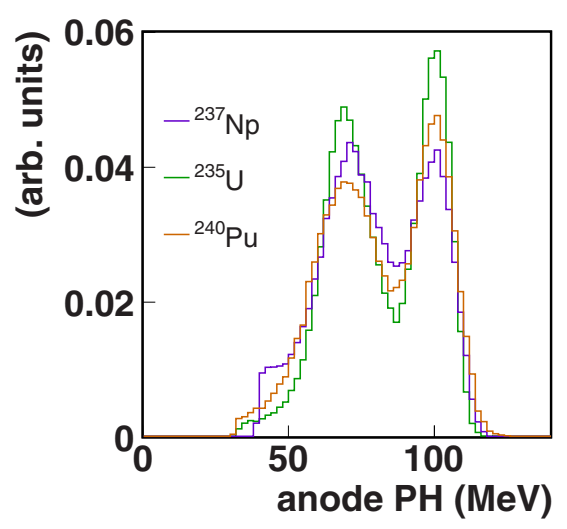

FIG. 4. (Color online) Pulse height (PH) distribution for the ${ }^{240} \mathrm{Pu}$ (orange), ${ }^{237} \mathrm{~Np}$ (violet), and ${ }^{235} \mathrm{U}$ (green) samples used in this experiment taken with $E_{n}=1.8 \mathrm{MeV}$ and using $\mathrm{CH}_{4}$ as counting gas. Higher fission fragment energy loss is seen at low $\mathrm{PH}$ values for the ${ }^{237} \mathrm{~Np}$ sample. Additionally, a degradation of the $\mathrm{PH}$ distribution is evident for the more active sample ${ }^{240} \mathrm{Pu}$, even though it is the thinnest one. 
where $t_{0}$ is the total thickness of the sample compound, $R$ is the FF range within the sample compound, $a_{2}$ is the anisotropy of the FF angular distribution for $E_{n}$, and

$$
\eta=\frac{\sqrt{\frac{E_{n}}{E_{f}} A_{f}}}{A_{n}+1}
$$

accounts for the momentum transfer from the neutron to the fissioning nucleus. The anisotropy $\left(a_{2}\right)$ was calculated experimentally from the cosine distributions obtained.

This correction is not higher than $2 \%$ in most of the cases. For the same isotope, it is always higher when the foil is placed downstream and for the highest neutron energy. When using the thickest sample $\left({ }^{237} \mathrm{~Np}\right)$, the correction goes up to $5 \%$ at a neutron energy of $3 \mathrm{MeV}$.

\section{Neutron spectrum, sample inhomogeneity, and solid angle}

Depending on the thickness of the neutron producing target, the energy width of the neutrons will vary. Since the proton-induced cross section does not take a constant value, this effect needs to be accounted for in the final cross section calculation by folding the reference cross section with the neutron energy distribution. In addition, the emission of neutrons at the producing target is not fully isotropic. The samples used in the experiment have different spot sizes and some are not homogeneous, thus the shape of the emitted flux, as a function of the solid angle, is used to correct for these differences. The differential cross section as a function of neutron emission angle for each reaction, ${ }^{7} \mathrm{Li}(p, n){ }^{7} \mathrm{Be}$ and $\mathrm{T}(p, n)^{3} \mathrm{He}$, was obtained from Refs. [25,26].

The influence of both effects is smaller than $0.05 \%$ when the two samples have the same spot size, goes up to $1.3 \%$ when their sizes are different and one of the samples is not homogeneous, and up to $2.5 \%$ when both samples are homogeneous having different diameter.

\section{Excited state of the ${ }^{7} \mathrm{Li}(p, n)^{7} \mathrm{Be}$ reaction}

The reaction to the first excited state of the ${ }^{7} \mathrm{Li}(p, n)^{7} \mathrm{Be}$ starts at $E_{n} \approx 0.7 \mathrm{MeV}$ and has around $0.4 \mathrm{MeV}$ lower energy than the reaction to the nuclear ground state. The differential cross section into the first excited state is, at least, one order of magnitude lower than into the ground state [25] and its corresponding correction is smaller than $1 \%$.

\section{Background neutrons.}

A nondesirable neutron background was generated through inelastic scattering on the material in between the neutron producing target and the sample deposits as well as by neutrons returning from the walls (room return). Two setup configurations were used during the experiments. The first one (setup \#1) consisted mainly of a neutron producing target cooled by $2 \mathrm{~mm}$ of water and, at $7 \mathrm{~cm}$ distance, the samples were placed at the center of the TFGIC. The second configuration (setup \#2) consisted of a water layer of $1 \mathrm{~mm}$ and, additionally, the TFGIC and part of the beam line were shielded by a paraffin- $\mathrm{B}_{4} \mathrm{C}$ wall (see Fig. 3).

To evaluate the neutron moderation on the different materials between the neutron source and the samples as well as the room-return effect, MCNP [23] simulations were performed for the two different configurations (see Fig. 5). The neutron energy distribution, its emission angle, and emission probability entered the simulations according to the information given in Refs. [25,26]. The result given by the simulation was the flux as a function of the neutron energy impinging on the sample deposits, i.e., at the center of the TFGIC, folded with the neutron-induced fission cross section of the isotope of interest: $\sum_{i} \Phi\left(E_{i}\right) \sigma\left(E_{i}\right)$. Because the goal was to determine the $(n, f)$ cross section at a single neutron energy, the flux at the neutron energy of interest folded with the $(n, f)$ cross section of the sample isotope, $\Phi\left(E_{n}\right) \sigma\left(E_{n}\right)$, was considered too. The ratio of these two quantities gives the correction factor to be applied at each neutron energy for each isotope. For all isotopes the $\sigma\left(E_{i}\right)$ considered was the one available in MCNP. This correction factor accounts for the number of fission events detected in the TFGIC that were not induced by neutrons of the energy of interest. The results for setup \#1 are presented in Fig. 6 for (a) a LiF neutron producing target and (b) a TiT neutron producing target.
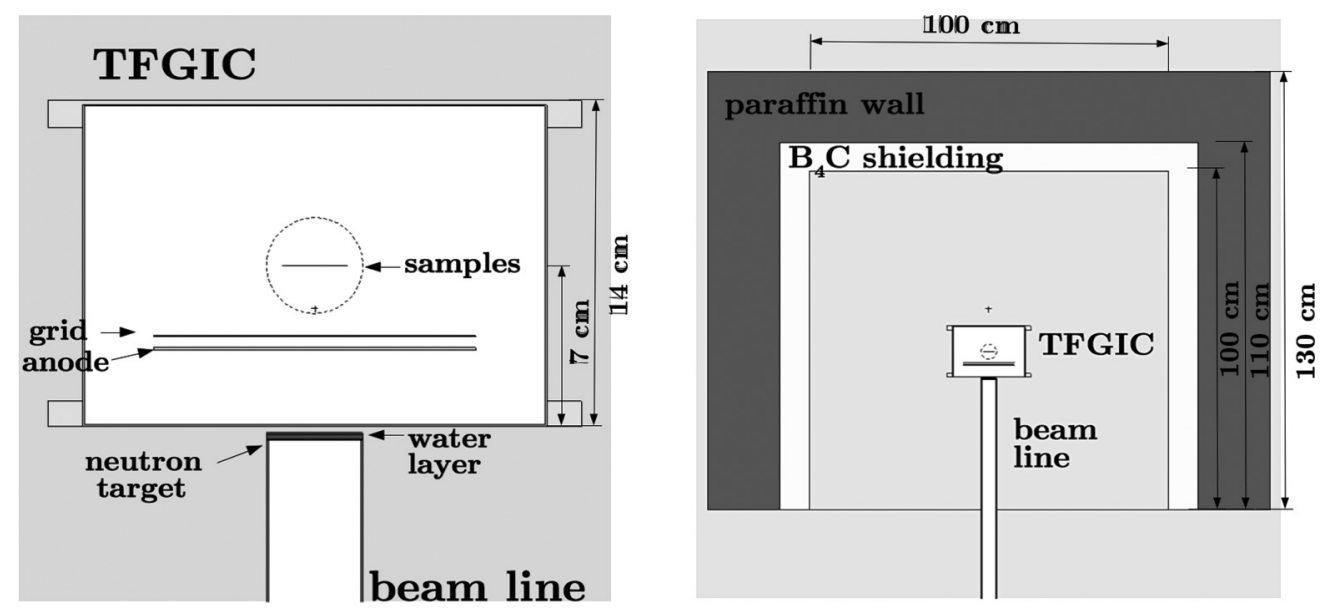

FIG. 5. Geometry used in the simulations with the MCNP code. Left: setup \#1; right: setup \#2. Besides the shielding, the main difference between the two setups is the thickness of the water layer (2 mm for setup \#1; $1 \mathrm{~mm}$ for setup \#2). 

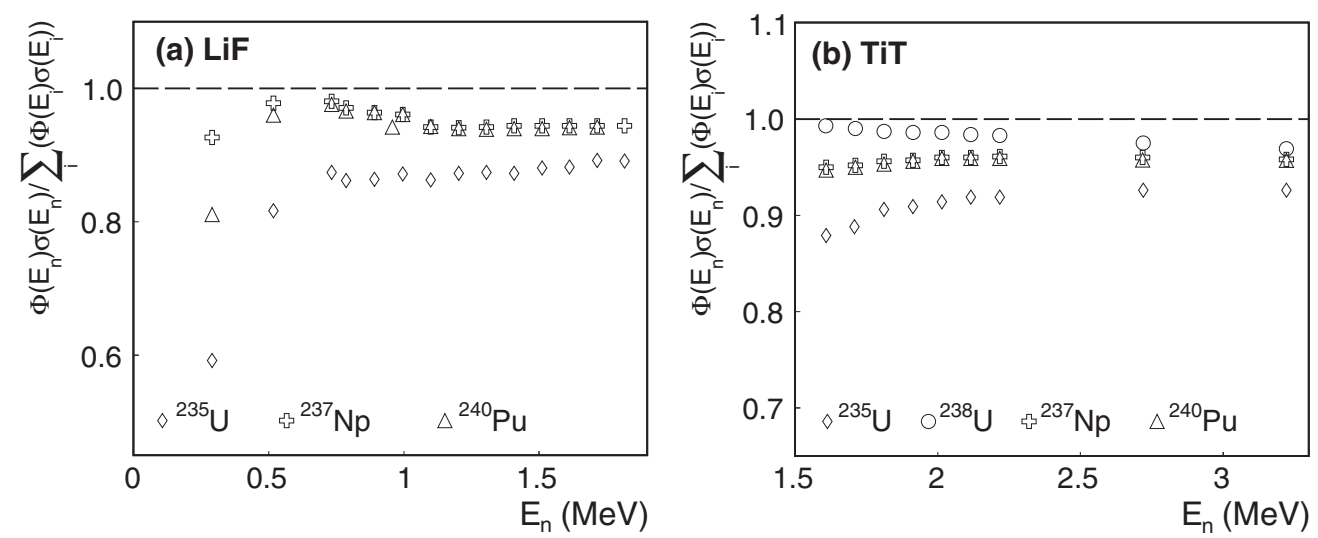

FIG. 6. Correction factors to account for the neutrons outside the region of interest produced by a thermalization in setup \#1 between the neutron producing target and the fissile deposits. The results given are the ratio of the flux at the neutron energy of interest folded with the $(n, f)$ cross section of the sample isotope considered $\left[\Phi\left(E_{n}\right) \sigma\left(E_{n}\right)\right]$ and the flux as a function of the neutron energy impinging the sample deposits folded with the neutron-induced fission cross section of the isotope of interest [ $\left.\sum_{i} \Phi\left(E_{i}\right) \sigma\left(E_{i}\right)\right]$. (a) Using a LiF neutron producing target; (b) using a TiT neutron producing target. For each case the initial neutron energy was a probability distribution as a function of the emission angle. The values were taken from Refs. [25,26].

It has been observed with the results of the simulations that the influence on the neutron spectrum of the room return due to the paraffin- $\mathrm{B}_{4} \mathrm{C}$ is nearly negligible (below $<0.6 \%$ ) (see Fig. 7). Consequently, the neutron thermalization taking place in the water layer becomes the major contribution to the degradation of the neutron spectrum. This degradation of the neutron spectrum will produce, for instance, a higher fission count rate on the ${ }^{235} \mathrm{U}$ side, due to the increase on the ${ }^{235} \mathrm{U}(n, f)$ cross section with decreasing neutron energy, leading to calculating a reduced cross section of the isotope of interest. In the case of samples with a fission threshold, when measuring at neutron energies close to the fission threshold the ratio is close to 1 if the $(n, f)$ cross section of the isotope becomes 0 at around 200-300 keV lower energy than the one to be measured. At the plateau region of the $(n, f)$ cross section $\left(1.2-1.8 \mathrm{MeV}\right.$ for ${ }^{237} \mathrm{~Np}$ and $\left.{ }^{240} \mathrm{Pu}\right)$ the correction factors do not reach 1 because the neutron energy spectra cover a region down to the fission threshold. In the neutron energy region where the TiT is employed, the strongest corrections are applied when the ${ }^{235} \mathrm{U}$ sample is used, because its cross section in this region does not show a flat behavior. For the samples with a fission threshold below $E_{n}=1.5 \mathrm{MeV}\left({ }^{237} \mathrm{~Np}\right.$ and ${ }^{240} \mathrm{Pu}$ ), the behavior is constant in the entire region. Finally, when using the ${ }^{238} \mathrm{U}$ sample (fission threshold at $E_{n}=1.6 \mathrm{MeV}$ ), the correction increases when approaching the plateau region of its $(n, f)$ cross section. Additionally, these correction factors cancel out when the two samples employed have a similar $(n, f)$ cross section shape.
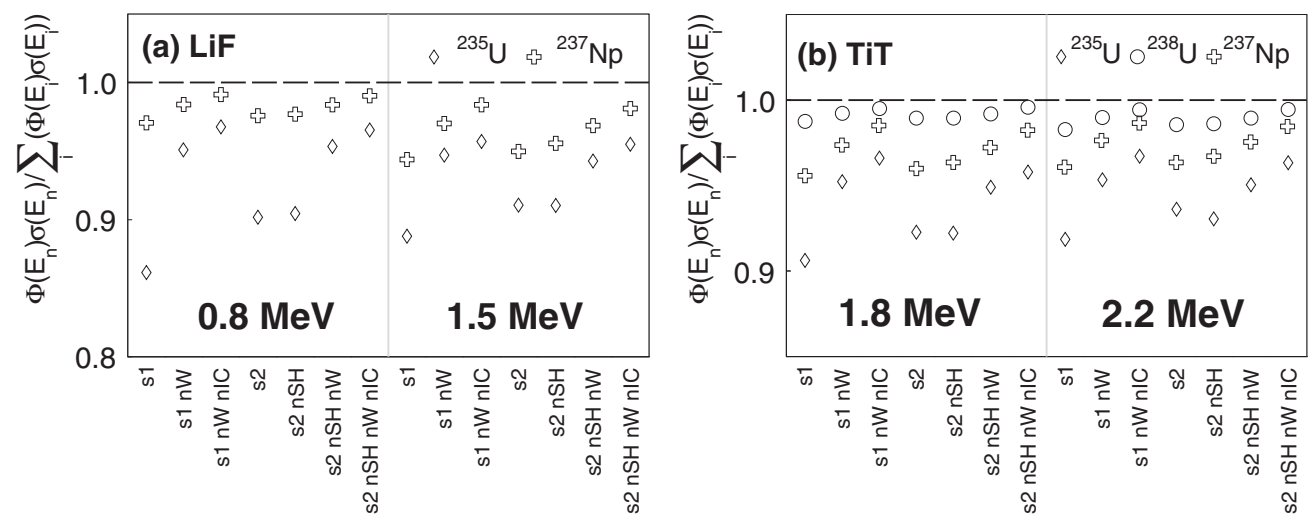

FIG. 7. Influence of the shielding, the water layer and the ionization chamber to the correction factors for each of the two setups for (a) $0.8 \mathrm{MeV} \mathrm{LiF}$ and 1.5 MeV LiF and (b) $1.8 \mathrm{MeV}$ TiT and 2.2 MeV TiT. Labels are s1 (setup \#1), s1 nW (setup \#1 without water layer), $\mathrm{s} 1 \mathrm{nW}$ nIC (setup \#1 without water layer and ionization chamber structure), s2 (setup \#2), s2 nSH (setup \#2 without shielding), s2 nSH nW (setup \#2 without shielding and water layer), and $\mathrm{s} 2 \mathrm{nSH} \mathrm{nW} \mathrm{nIC} \mathrm{(setup} \mathrm{\# 2} \mathrm{without} \mathrm{shielding,} \mathrm{water} \mathrm{layer,} \mathrm{and} \mathrm{ionization} \mathrm{chamber} \mathrm{structure).}$ For a better visualization the ${ }^{240} \mathrm{Pu}$ correction factors are not shown since its cross section is very similar to the one of ${ }^{237} \mathrm{~Np}$. In the case of LiF only the correction factors of ${ }^{235} \mathrm{U}$ and ${ }^{237} \mathrm{~Np}$ are presented, since no measurements were performed with ${ }^{238} \mathrm{U}$. Noticeably, for the two setups, the biggest influence comes from the water layer (2 mm for setup \#1 and $1 \mathrm{~mm}$ for setup \#2); on the other hand, the shielding structure of setup $\# 2$ has a negligible influence on the neutron background. Additionally, it is clear that isotopes with a fission threshold at higher energies are less affected by the neutron background than fissile isotopes. 
TABLE II. Summary of the systematic uncertainties associated with the fission cross section measurements.

\begin{tabular}{lc}
\hline \hline Uncertainty source & \\
\hline Statistical & $0.5 \%$ \\
Counts SF & $<1.1 \%$ \\
${ }^{240} \mathrm{Pu}$ Mass & $0.4 \%$ \\
${ }^{237} \mathrm{~Np}$ Mass & $0.3 \%$ \\
${ }^{235} \mathrm{U}$ Mass & $1.5-2 \%$ \\
${ }^{238} \mathrm{U}$ Mass & $0.5 \%$ \\
Efficiency & $1 \%$ \\
Sample purity & $0.001 \%$ \\
Correction of neutron spectrum & $<0.2 \%$ \\
MCNP correction of the thermalized flux (ratio) & $0.5 \%$ \\
${ }^{237} \mathrm{~Np}$, ENDF evaluation & $2.2-4 \%$ \\
${ }^{238} \mathrm{U}$, standard [27] & $0.7 \%$ \\
${ }^{235} \mathrm{U}$, standard [27] & $<0.8 \%$ \\
\hline \hline
\end{tabular}

\section{B. Sources of uncertainty}

A detailed study of the uncertainties related to each correction and value used has been done. As previously described, all samples used for this experiment were remeasured in order to lower the uncertainty associated with their mass. The other factor that contributes to an increase of the uncertainty is the efficiency. As discussed in Ref. [16], the related uncertainty on the efficiency for samples with very high $\alpha$ activity, such as the one of ${ }^{240} \mathrm{Pu}$, could not be predicted better than $1 \%$. Table II lists all the uncertainties related to this experiment.

In the case of achieving absolute cross section values, the total uncertainty for each measurement is smaller than $3 \%$ in cases where the reference cross section was ${ }^{235} \mathrm{U}(n, f)$ or ${ }^{238} \mathrm{U}(n, f)$. In the case of the normalization with the ${ }^{237} \mathrm{~Np}(n, f)$ cross section the total uncertainty is close to $5 \%$, because the reference sample is not a standard. The statistical uncertainty is, in most of the individual data sets, around $0.5 \%$, except for a single case where it amounts to $1.4 \%$.

\section{Results}

Measuring the ${ }^{240} \mathrm{Pu}(n, f)$ cross section relative to a secondary standard [i.e., $\left.{ }^{238} \mathrm{U}(n, f)\right]$ or even not a standard [i.e., $\left.{ }^{237} \mathrm{~Np}(n, f)\right]$ resulted in deeper knowledge of these two cross sections. Because of that, those two isotopes were studied in terms of their neutron-induced fission cross section relative to ${ }^{235} \mathrm{U}(n, f)$. First of all, the ${ }^{237} \mathrm{~Np}(n, f)$ cross section was benchmarked using the ${ }^{235} \mathrm{U}(n, f)$ cross section and the results obtained were in agreement within $2 \%$ with the ENDF/B-VII. 1 evaluation in the range of interest $\left(E_{n}=[0.5,1.8] \mathrm{MeV}\right.$, and using the ${ }^{7} \mathrm{Li}(p, n){ }^{7}$ Be reaction). The results obtained using the ${ }^{238} \mathrm{U}(n, f)$ cross section as reference need to be normalized by the ${ }^{238} \mathrm{U}(n, f) /{ }^{235} \mathrm{U}(n, f)$ ratio due to a misbehavior of the ${ }^{238} \mathrm{U}$ sample. Therefore, a normalization needed to be applied when the measured ratio was ${ }^{240} \mathrm{Pu}(n, f) /{ }^{238} \mathrm{U}(n, f)$ :

$$
\sigma_{240 \mathrm{Pu}}^{\exp }\left(E_{n}\right)=\frac{\sigma_{240 \mathrm{Pu}}^{\exp }\left(E_{n}\right)}{\sigma_{238 \mathrm{U}}^{\exp }\left(E_{n}\right)} \frac{\sigma_{238 \mathrm{U}}^{\exp }\left(E_{n}\right)}{\sigma_{235 \mathrm{U}}^{\exp }\left(E_{n}\right)} \times \sigma_{235 \mathrm{U}}^{\mathrm{ENDF}}\left(E_{n}\right),
$$

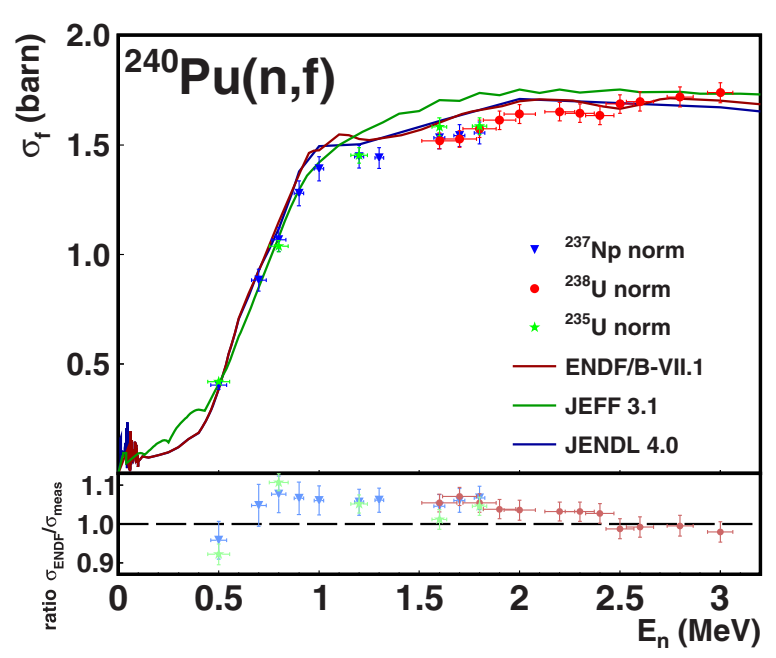

FIG. 8. (Color online) Neutron-induced fission cross section of ${ }^{240} \mathrm{Pu}$ using as reference ${ }^{237} \mathrm{~Np}(n, f)$ (blue triangles), ${ }^{235} \mathrm{U}(n, f)$ (green stars), and ${ }^{238} \mathrm{U}(n, f)$ (red dots). The agreement of the three data sets is striking, especially at 1.6 and $1.8 \mathrm{MeV}$ incoming neutron energy. Yet, the ratio plot shows a deviation of 3-7\% in the neutron energy range from $0.7 \mathrm{MeV}$ up to $2.5 \mathrm{MeV}$ compared with the ENDF/B-VII.1 evaluation.

where the index "exp" corresponds to experimental data and the index "ENDF" to the value of the ENDF/B-VII.1 evaluation.

The results obtained for each of the three measured ratios are compared in Fig. 8, where all the data is normalized to the respective ENDF/B-VII.1 evaluations, except for the ratio to ${ }^{238} \mathrm{U}(n, f)$ where the normalization of Eq. (4) is applied. When using the ${ }^{7} \mathrm{Li}(p, n)^{7} \mathrm{Be}$ neutron producing target, i.e., below $1.8 \mathrm{MeV}$, the values obtained with the ${ }^{237} \mathrm{~Np}(n, f)$ normalization (blue triangles) are in perfect agreement with the ones obtained using the ${ }^{235} \mathrm{U}(n, f)$ (green stars). Additionally, at the fission threshold the results resemble the current evaluations, especially the JEFF 3.1. From $1 \mathrm{MeV}$ up to $1.8 \mathrm{MeV}$, this experiment is up to $7 \%$ lower than the ENDF/B-VII.1 evaluation, as can be seen from the ratio plot in the lower part of Fig. 8. Above 1.6-1.8 MeV, when the $\mathrm{T}(p, n)^{3} \mathrm{He}$ reaction is used, the data presented as normalized to the ${ }^{238} \mathrm{U}(n, f)$ cross section (red dots) were in addition renormalized using Eq. (4). An outstanding agreement is obtained at $1.6 \mathrm{MeV}$ and $1.8 \mathrm{MeV}$ with the other two normalizations. Further, the fission cross section shape is very well maintained, yet with 3-5\% lower values for incoming neutron energies up to $2.5 \mathrm{MeV}$. For energies above $2.5 \mathrm{MeV}$, the cross section is increasing compared with the prediction by any of the three evaluations. The reason for this effect is that the normalization ratio ${ }^{238} \mathrm{U}(n, f) /{ }^{235} \mathrm{U}(n, f)$ was just measured up to $2.8 \mathrm{MeV}$. Thus, the extrapolated ratio at $3.0 \mathrm{MeV}$ obtained for normalization purposes might be overestimated.

A weighted average was calculated for all subsets of data; the results are shown in Fig. 9 together with previous experimental values and the most recent evaluations. The neutron energy window chosen for each weighted data point was the wider window from the individual data points to 


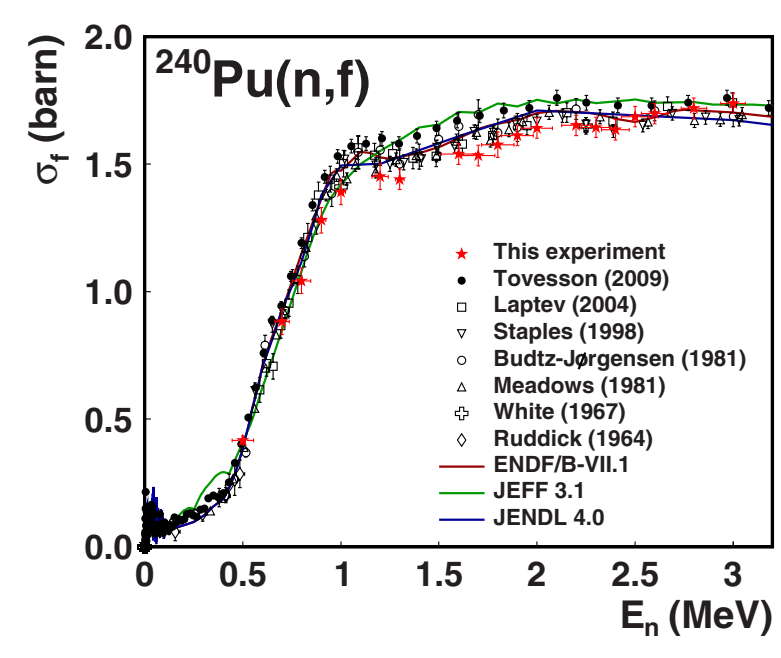

FIG. 9. (Color online) Summary of the results of this experiment (red stars) compared with the most relevant experiments performed on the neutron-induced fission cross section of ${ }^{240} \mathrm{Pu}$ and with current evaluations. The evaluations chosen are: ENDF/B-VII.1 [12], JEFF 3.1 [11], and JENDL 4.0 [13]. The experimental data shown are Ruddick and White (1964) [5] (open diamonds), White (1967) [6] (open crosses), Meadows (1981) [7] (open triangle up), Budtz-Jørgensen and Knitter (1981) [8] (open circles), Staples and Morley (1998) [9] (open triangles down), Laptev et al. (2004) [10] (open squares), and Tovesson et al. (2009) [4] (full circles). Selected data are shown for legibility of the plot. Data found as a ratio to ${ }^{235} \mathrm{U}(n, f)$ were normalized to the ENDF/B-VII. 1 evaluation of this isotope. Further explanation is given in the text.

be weighted. The uncertainty presented is the larger of the individual data points used to calculate the weighted average.

An agreement is seen at the threshold region, in particular with the JEFF 3.1 evaluation. At the plateau region, this experiment confirms the lower set of values available in
EXFOR, i.e., those from Laptev et al. (2004) [10] and Staples and Morley (1998) [9].

\section{CONCLUSIONS}

The neutron-induced fission cross section of ${ }^{240} \mathrm{Pu}$ has been measured in the neutron energy range from $0.5 \mathrm{MeV}$ up to $3 \mathrm{MeV}$, following the high-priority request from the OECD-NEA. The neutrons were produced via two protoninduced reactions, namely ${ }^{7} \mathrm{Li}(p, n)^{7} \mathrm{Be}$ and $\mathrm{T}(p, n)^{3} \mathrm{He}$, at the Van de Graaff accelerator at JRC-IRMM. A twin Frisch-grid ionization chamber was used as fission fragment detector. Three reference samples were used in the experimental campaigns: ${ }^{237} \mathrm{~Np}(n, f),{ }^{238} \mathrm{U}(n, f)$, and ${ }^{235} \mathrm{U}(n, f)$. Additionally, the ${ }^{238} \mathrm{U}(n, f)$ and the ${ }^{237} \mathrm{~Np}(n, f)$ cross sections were benchmarked using ${ }^{235} \mathrm{U}(n, f)$. The ${ }^{240} \mathrm{Pu}(n, f)$ cross section obtained with any of the reference samples at $1.6-1.8 \mathrm{MeV}$ is in agreement within uncertainties. At the fission threshold, the ${ }^{240} \mathrm{Pu}(n, f)$ cross section is in agreement not only with previous experimental data but also with the JEFF 3.1 evaluation. Above $1 \mathrm{MeV}$, the data extracted when doing a weighted average of all subsets of data are around 3-5\% lower than the ENDF/B-VII.1 evaluation, but in very good agreement with the recent data sets from Laptev et al. (2004) [10] and Staples and Morley (1998) [9].

The main uncertainty contribution was coming from the mass of the samples, yet an effort was done to re-measure this quantity for all the samples used. Additionally, the efficiency was considered to have an uncertainty of $1 \%$. Finally, when doing the normalization, the highest uncertainty contribution came from the ${ }^{237} \mathrm{~Np}(n, f)$ cross section, which currently is not known to better than $2.2-4 \%$.

\section{ACKNOWLEDGMENTS}

One of the authors (P.S.-C.) acknowledges financial support from the ANDES Collaboration (Contract No. FP7-249671).
[1] M. Salvatores, Uncertainty and Target Accuracy Assessment for Innovative Systems Using Recent Covariance Data Evaluations (NEA/WPEC-26) (Nuclear Energy Agency (OECD), Paris, 2008).

[2] ANDES project, http://www.andes-nd.eu

[3] Experimental Nuclear Reaction Data (EXFOR), http://www.nndc.bnl.gov/exfor/exfor.htm

[4] F. Tovesson, T.S. Hill, M. Mocko, J.D. Baker, and C.A. McGrath, Phys. Rev. C 79, 014613 (2009).

[5] P. Ruddick and P. White, J. Nucl. Energy 18, 561 (1964).

[6] P. White, J. Nucl. Energy 21, 671 (1967).

[7] J. Meadows, Nucl. Sci. Eng. 79, 233 (1981).

[8] C. Budtz-Jørgensen and H.-H. Knitter, Nucl. Sci. Eng. 79, 380 (1981).

[9] P. Staples and K. Morley, Nucl. Sci. Eng. 129, 149 (1998).

[10] A. Laptev, A. Y. Donets, A. Fomichev, A. Fomichev, R. Haight, O. Shcherbakov, S. Soloviev, Y. V. Tuboltsev, and A. Vorobyev, Nucl. Phys. A 734, E45 (2004).
[11] A. Santamarina, D. Bernard, P. Blaise, M. Coste, A. Courcelle, T. Huynh, C. Jouanne, P. Leconte, O. Litaize, S. Mengelle et al., JEFF Report 22, 2 (2009).

[12] M. B. Chadwick, M. Herman, P. Obložinský, M. E. Dunn, Y. Danon, A. C. Kahler, D. L. Smith, B. Pritychenko, G. Arbanas, R. Arcilla et al., Nucl. Data Sheets 112, 2887 (2011).

[13] K. Shibata, O. Iwamoto, T. Nakagawa, N. Iwamoto, A. Ichihara, S. Kunieda, S. Chiba, K. Furutaka, N. Otuka, T. Ohasawa et al., J. Nucl. Sci. Technol. 48, 1 (2011).

[14] C. Budtz-Jørgensen, H.-H. Knitter, C. Straede, F.-J. Hambsch, and R. Vogt, Nucl. Instrum. Methods Phys. Res. Sect. A 258, 209 (1987).

[15] A. Al-Adili, F.-J. Hambsch, S. Oberstedt, S. Pomp, and S. Zeynalov, Nucl. Instrum. Methods Phys. Res. Sect. A 624, 684 (2010).

[16] P. Salvador-Castiñeira, T. Bryś, R. Eykens, F.-J. Hambsch, A. Moens, S. Oberstedt, G. Sibbens, D. Vanleeuw, M. Vidali, and C. Pretel, Phys. Rev. C 88, 064611 (2013).

[17] C++, http://www.cplusplus.com/ 
[18] ROOT, http://root.cern.ch/drupal/

[19] G. Sibbens, A. Moens, R. Eykens, D. Vanleeuw, F. Kehoe, H. Kühn, R. Wynants, J. Heyse, A. Plompen, R. Jakopiĉ, S. Richter, and Y. Aregbe, J. Rad. Nucl. Chem. 299, 1093 (2014).

[20] S. Pommé (private communication).

[21] A. Göök (private communication).

[22] R. Van Ammel (private communication).

[23] MCNP, https://mcnp.lanl.gov/
[24] G. W. Carlson, Nucl. Instrum. Methods 119, 97 (1974).

[25] H. Liskien and A. Paulsen, At. Data Nucl. Data Tables 11, 569 (1973).

[26] H. Liskien and A. Paulsen, At. Data Nucl. Data Tables 15, 57 (1975).

[27] A. D. Carlson, V. G. Pronyaev, D. L. Smith, N. M. Larson, Z. Chen, G. M. Hale, F.-J. Hambsch, E. V. Gai, S.-Y. Oh, S. A. Badikov et al., Nucl. Data Sheets 110, 3215 (2009). 